(C) [2008] IEEE. Reprinted, with permission, from [Steven W. Suzy, Branko G. Cellerz, Andrey V. Savkinz, Hung T. Nguyeny,Teddy M. Chengz, Ying Guo and Lu Wang, Portable sensor based dynamic estimation of human oxygen uptake via nonlinear multivariable modelling, Engineering in Medicine and Biology Society, 2008. EMBS 2008. 30th Annual International Conference of the IEEE 20-25 Aug. 2008]. This material is posted here with permission of the IEEE. Such ermission of the IEEE does not in any way imply IEEE endorsement of any of the University of Technology, Sydney's products or services. Internal or personal use of this material is permitted. However, permission to reprint/republish this material for advertising or promotional purposes or for creating new collective works for resale or redistribution must be obtained from the IEEE by writing to pubs-permissions@ieee.org. By choosing to view this document, you agree to all provisions of the copyright laws protecting it 


\title{
Portable sensor based dynamic estimation of human oxygen uptake via nonlinear multivariable modelling
}

\author{
Steven W. Su ${ }^{\ddagger \dagger}$, Branko G. Celler ${ }^{\ddagger}$, Andrey V. Savkin ${ }^{\ddagger}$, Hung T. Nguyen ${ }^{\dagger}$, \\ Teddy M. Cheng ${ }^{\ddagger}$, Ying Guo ${ }^{\star}$, and Lu Wang $\ddagger$
}

\begin{abstract}
Noninvasive portable sensors are becoming popular in biomedical engineering practice due to its ease of use. This paper investigates the estimation of human oxygen uptake $\left(\mathrm{VO}_{2}\right)$ of treadmill exercises by using multiple portable sensors (wireless heart rate sensor and triaxial accelerometers). For this purpose, a multivariable Hammerstein model identification method is developed. Well designed PRBS type of exercises protocols are employed to decouple the identification of linear dynamics with that of nonlinearities of Hammerstein systems. The support vector machine regression is applied to model the static nonlinearities. Multivariable ARX modelling approach is used for the identification of dynamic part of the Hammerstein systems. It is observed the obtained nonlinear multivariable model can achieve better estimations compared with single input single output models. The established multivariable model has also the potential to facilitate dynamic estimation of energy expenditure for outdoor exercises, which is the next research step of this study.
\end{abstract}

Index Terms-Oxygen uptake; Multivariable Hammerstein model; Identification; Support Vector Regression; Treadmill exercise.

\section{INTRODUCTION}

O XYGEN uptake is an important physiological parameter for the determination of functional health status and clinical assessments in normal and pathological conditions. The main purpose of the paper is to explore dynamic estimation of oxygen uptake by using multiple noninvasive portable sensors. The potential application areas of this study include but not limited to training monitoring for elite athletes, health monitoring of patients with diabetes, and remote health assessment of elderly patients in telemedicine.

Recently, practical portable sensors such as Triaxial Accelerometers (TAs), pedometers, and heart rate (HR) sensors are used for the estimation of oxygen uptake during exercises [1]. These sensors provide a potentially portable platform for the reliable estimation of oxygen uptake under sensor failure or malfunctions which are often encountered for wireless portable sensors. An example of these sensor failures for a wireless HR sensor is the artefacts generated by high impedance, body movements, and sudden disconnections. From data fusion and fault tolerance point of view, the combination of sensors with different measuring mechanisms has many advantages [2].

$\dagger$ Key University Research Centre for Health Technologies, The Faculty of Engineering, University of Technology, Sydney, Australia.

$\ddagger$ Human Performance Group, Biomedical Systems Lab, School of Electrical Engineering \& Telecommunications University of New South Wales,UNSW Sydney N.S.W. 2052 Australia.

$\star$ Autonoumous Systems Lab, CSIRO ICT Center, Australia.

This research was supported by the Australian Research Council.

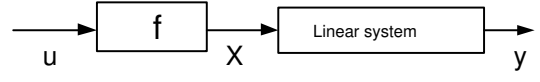

Fig. 1. A Hammerstein system.

Based on this consideration, this paper selects Polar wireless HR sensor and TAs to estimate $V O_{2}$. TAs can evaluate the energy expenditure directly associated with body movement, and facilitate temporal tracking of the frequency, intensity, and duration of activity. On the other hand, HR is a measurement of body response to exercise. These two kinds of sensors are therefore quite different in measurement mechanism. They have their own disadvantages as well. HR reflects the relative stress placed on the cardiopulmonary system due to activity [3], but it can also be elevated by emotional stress, which is independent of any change in oxygen uptake [1]. TAs measures exercise density but cannot assess body responses. The combination of these two kinds of sensors can well remedy these shortcomings. Furthermore, these sensors are cost effective portable sensors and easy to use. Based on the above consideration, we select HR and TAs to estimate $\mathrm{VO}_{2}$ dynamically.

For the modelling of oxygen uptake, relevant papers in current literature have mainly concentrated on either steady state prediction [4] [5] or dynamical estimation of onset and offset exercise [6] [7]. Paper [8] presented a novel SISO Hammerstein modeling method to depict both steady state and transient of oxygen uptake. This model can be described as a static nonlinear block followed by a dynamic linear system (see Fig.1).

The dynamical estimation of oxygen uptake in paper [8] is based on the information of walking speed which is obtained from the motor speed of treadmill machine and is not applicable for free-living conditions. In this study, TAs combined with wireless HR sensor are used to dynamically estimate $\mathrm{VO}_{2}$ as the replacement of the recording of walking speed. We also extend the SISO Hammerstein modeling method in [8] [9] to multivariable case in order to cope with multiple sensor based estimation. As stated in [8] for SISO modelling, the extended multivariable Hammerstein model identification approach is also quite suitable for the identification of oxygen uptake model. Due to the total decoupling of static part identification (based on steady state experiment data) and dynamic part identification (based on dynamic experiment data), it is possible to apply different measurement approaches for steady state tests and dynamic 


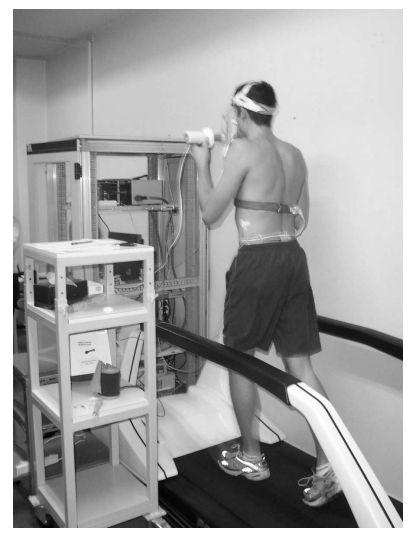

Fig. 2. A typical experimental senario.

experiments respectively.

In this paper, we only estimate $V O_{2}$ for moderate exercises (less than $100 \mathrm{w}$ ). We believe when the $\mathrm{VO}_{2}$ estimation is in a relative wide range, the nonlinear modeling approach would be much more effective than linear one.

This paper is organized as follows. The extended multivariable Hammerstein model identification method and its associated experimental arrangements are introduced in Section II. Modelling results are summarized in Section III. Section IV gives conclusions.

\section{METHOD}

\section{A. Subjects}

Six normal male subjects (aged $28 \pm 5.5 \mathrm{yr}$, height 176 $\pm 5 \mathrm{~cm}$, body weight $70 \pm 11 \mathrm{~kg}$ ) attend the experiments. They are all active, but do not participate in formal training or organized sports. All the subjects knew the protocol and the potential risks, and had given their informed consent. The protocol was approved by the Ethics Committee of the University of New South Wales.

\section{B. Instrumentation}

Heart rate was monitored beat by beat using a wireless Polar system. The core part of accelerometer was ADXL210 (Analog Device, Inc.), a piezoresistive accelerometer supplied by Analog Electronics. The ADXL210 has a range of $\pm 10 \mathrm{~g}$, a frequency range of $0-50 \mathrm{~Hz}$, an RMS noise estimate of $4.33 \times 10^{-3} \mathrm{~g}$ and a peak-to-peak noise estimate of $17.2 \times 10^{-3}$. Ventilation and pulmonary exchange were measured on a breath by breath basis. Minute ventilation was measured during inspiration using a Turbine Flow Transducer model K520-C521 (Applied Electrochemistry, USA). Pulmonary gas exchange was measured using S-3A and CD3A gas analyzers (Applied Electrochemistry, USA). Before each individual exercise test, the turbine meter was calibrated using a 3.0 liters calibration syringe.

\section{Procedure}

A typical experimental senario is shown in Fig.2. All experiments were conducted in the afternoon, and the subjects were permitted to have a light meal one hour before measurements were recorded. Initially, the subjects were asked to walk for about 10 minutes on the treadmill to familiarize themselves with the experiment. The subjects were then requested to walk at six levels of different speeds $(2,3,4,5,6$ and $7 \mathrm{~km} / \mathrm{h})$. Each level took a total period of 5 minutes, and was followed by a 10 -minute resting period. The oxygen uptake was recorded and averaged every two minutes by using a mixing chamber based gas analysis and ventilation measurement system (Applied Electrochemistry, USA). Finally, in order to identify linear dynamic part of the Hammerstein system, subjects were also requested to walk on the treadmill under a PRBS input. Throughout the experiments, the breath by breath tidal volume and the concentration of oxygen were recorded to calculate breath by breath oxygen uptake. The outputs of TAs were also recorded.

\section{Multivariable modelling}

1) SVR based nonlinearity identification method: In [10], Bai showed that the identification of linear part of a Hammerstein model can be decoupled from nonlinear part with the help of the PRBS input. The reason is any static nonlinearity can be exactly characterized by a linear function under PRBS input which has the binary nature. In this study, the PRBS input is also employed. Thus, the identification of Hammerstein model can be obtained by the identification of static nonlinearity and linear dynamic separately.

For the identification of the nonlinearity, the so called $\epsilon$-insensitivity Support vector regression will be employed, which is convex and very efficient in terms of speed and complexity. The description of SVR regression is omitted due to space limitation. However, brief introduction of SVR regression can be found in papers [9] [8]. Details about SVR, such as the selection of radius $\epsilon$ of the tube, kernel function, and the regularization constant $C$, can be found in [11] [12].

2) Multivariable ARX modelling: The general structure of a SISO discrete time ARX model can be described as follows:

$$
A(q) y(t)=B(q) u\left(t-n_{k}\right)+e(t),
$$

where $u(t), y(t)$ and $e(t)$ are input, output and noise respectively, and

$$
\left\{\begin{array}{l}
A(q)=1+a_{1} q^{-1}+\cdots+a_{n_{a}} q^{-n_{a}} \\
B(q)=1+b_{1} q^{-1}+\cdots+b_{n_{b}} q^{-n_{b}} .
\end{array}\right.
$$

Models with two inputs and one output is shown as follows:

$$
A(q) y(t)=B_{1}(q) u\left(t-n_{k_{1}}\right)+B_{2}(q) u\left(t-n_{k_{2}}\right)+e(t),
$$

where:

$$
B_{i}(q)=1+b_{i 1} q^{-1}+\cdots+b_{n_{b i}} q^{-n_{b i}}, \quad i \in\{1,2\} .
$$

If model structure is predetermined the parameters of the model can be identified by using the least squares method. 


\section{DATA PRE-PROCESSING AND MULTIVARIABLE MODELLING}

\section{A. Data pre-processing}

The main data pre-processing task is related with TAs. The wireless portable TAs that were used are piezoresistive accelerometers. They are particularly suitable for detection of human movement due to their sensitivity to very low frequencies. The TAs were attached to the lower back close to the subject's centre of gravity. Accelerations were measured in a body-fixed axis system with measurement directions in antero-posterior (x), medio-lateral (y), and vertical (z). Individual outputs from the three measurements are high pass $(0.11 \mathrm{~Hz})$ and low-pass $(20 \mathrm{~Hz})$ filtered outputs to suppress DC-response and high frequencies that cannot be expected to arise from human movement. Filtered acceleration signals were processed to produce accelerometer output variables $I A A_{x}, I A A_{y}, I A A_{z}$ and $I A A_{t o t}$ :

$$
\left\{\begin{array}{ll}
I A A_{i} & =\frac{1}{T} \int_{t=0}^{T}\left|a_{i}\right| d t, \\
I A A_{t o t} & =I A A_{x}+I A A_{y}+I A A_{z},
\end{array} \quad i \in\{x, y, z\},\right.
$$

where $T=5$ seconds. For steady state experimental data, acceleration signals from the last 2-minute interval at the end of each walking stage were processed for steady state analysis.

\section{B. Modelling of static nonlinearity}

For the prediction of steady state oxygen uptake, book [5] proposed a famous linear static model to approximately estimate oxygen uptake in a given walking speed ranges. Papers [4] provided simple static nonlinear (polynomial) models. However, these models need walking speed information which is hard to be measured accurately in free living conditions. This study investigates the possibility of applying TAs and HR wireless portable sensors to estimate oxygen uptake in free living condition. We establishes not only SISO (single input single output) model ( $\mathrm{VO}_{2}$ vs $\mathrm{HR}$, and $\mathrm{VO}_{2}$ vs TAs), but also a MISO model ( $\mathrm{VO}_{2}$ vs HR\&TAs) based on SVR. The estimation results are shown in Fig.3-5 and summarized in Table I. In Fig. 3 and 4, the continuous curve stands for the estimated input output steady state relationship. The dotted lines indicate the $\epsilon$-insensitivity tube. The plus markers are the points of input and output data. The circled plus markers are the support points. In Fig.5, the solid circle stands for the estimated value and solid arrow stands for the measured value.

In terms of curve fitting results (training error in MSE) of these models, SVR regression is generally better than linear regression (LR). The best SVR estimation is the multivariable model $(\mathrm{MSE}=2.1)$. TAs based estimation $(\mathrm{MSE}=3.0)$ is less accurate than multivariable model, but is much better than HR sensor based modle (MSE=7.5). However, at this stage, it is still early to say that multivariable model is better than SISO models or TAs based model is better than HR based model. More experiments are needed to validate the identified models. Furthermore, as discussed before, the HR sensor is a measurement of body response

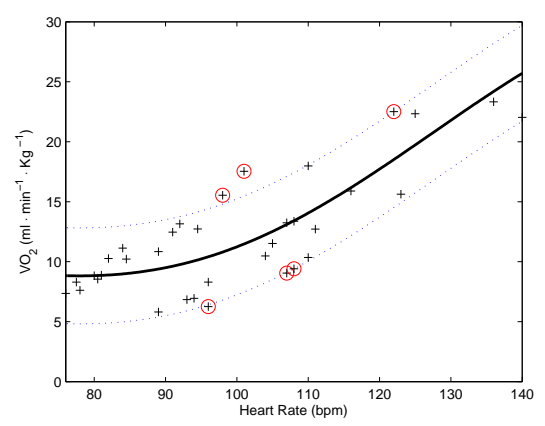

Fig. 3. SVR regression for $\mathrm{VO}_{2}$ estimation based on $\mathrm{HR}$ signal.

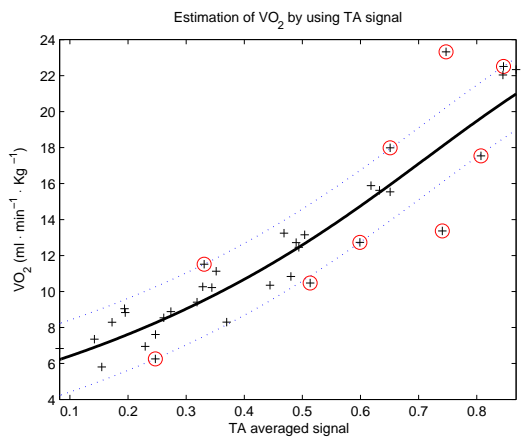

Fig. 4. SVR regression for $\mathrm{VO}_{2}$ estimation based on TA signal.

while TAs only measures body movement. The real oxygen uptake with same body movement may vary with individual body condition and environmental conditions (e.g. walking exercise in different incline and road surface in outdoor exercises).

Another interesting observation is that outputs of TAs and $\mathrm{HR}$ are correlated in a certain extend. That is possible a reason that in some restricted environmental condition and normal individuals using either TA or HR model can still achieve desired oxygen uptake estimation. Therefore, we present three models (not only a multivariable model) for the estimation of oxygen uptake in different situations.

\section{Modelling of dynamical part}

In order to avoid this coupling error, a well designed PRBS input is implemented in the automated treadmill system [8]. A PRBS has two levels $(a+$ and $a-)$ and switches from one
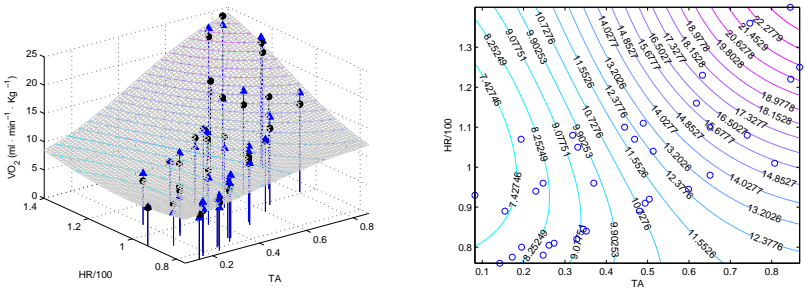

Fig. 5. SVR regression for $\mathrm{VO}_{2}$ estimation based on TA and HR signals. 


\begin{tabular}{lccc}
\hline \hline Relation & $\mathrm{VO}_{2}$ vs HR & $\mathrm{VO}_{2}$ VS TA & $\mathrm{VO}_{2}$ VS (TA \& HR) \\
\hline LR error & 8.9 & 3.4 & 3.1 \\
\hline SVR error & 7.5 & 3.0 & 2.1 \\
\hline
\end{tabular}

TABLE I

Estimation Mean SQUare ERRoR $\left(\left(m l \cdot m i n^{-1} K g^{-1}\right)^{2}\right)$ OF MODELS

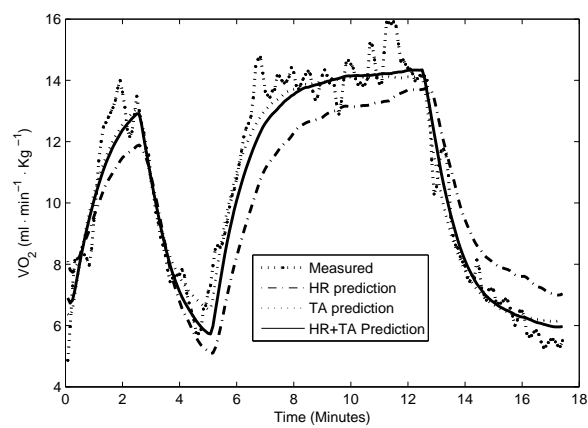

Fig. 6. Dynamic estimation of $\mathrm{VO}_{2}$.

level to the other at constant time intervals $\Delta$. It is periodic with period $T=\Delta N$, where $N=2 n-1$ and $n$ is an integer. In order to avoid nonlinear behaviour, the difference of the two levels of PRBS should be as close as possible. However, it is also required that the output responses under these two levels of inputs should be noticeably different (good signal to noise ratio) to ensure a reasonable parameter estimation results. For the selection of $\Delta$ and $N$, we need to compromise with the complexity of the selected model, response time of the system, noise level, and the total experimental time which the subjects can tolerate. In this study, we select $a+=6 \mathrm{~km} / \mathrm{h}, a-=4 \mathrm{~km} / \mathrm{h}, N=7$ and $\Delta=150$ seconds after several pre-experiments and detailed analysis of the modelling output.

Papers [13] [14] [15] often select first order exponential, with no time delays to describe the dynamics of oxygen uptake. In our previous study [8], it is also confirmed that the exponential rise in oxygen uptake directly reflects the rate of rise and drop in leg muscle oxygen uptake at the onset and offset of exercises. Therefore, we select first order ARX model (without delay) for the estimation of oxygen uptake. Based on the averaged data of PRBS input experiments, we identified the parameters of three individual models by using Matlab Identification Toolbox. The prediction results are shown in Figure 6 and summarized in Table II. In terms of the Akaike Final Prediction Error (FPE), the best prediction for $V O_{2}$ transient estimation is the multivariable ARX model ( $\mathrm{VO}_{2}$ vs $T A s \& H R$ ). Fig.6 shows that fairly good transient predictions are obtained.

\section{CONCLUSION}

In this study, we aim to estimate oxygen uptake dynamically under free living conditions by using portable wireless sensors. We extended the Hammerstein model identification

\begin{tabular}{|c|c|c|c|}
\hline Relation & $V \mathrm{O}_{2}$ vs $\mathrm{HR}$ & $V \mathrm{O}_{2}$ vs TA & $V \mathrm{O}_{2}$ vs (TA \& HR) \\
\hline FPE & 0.16 & 0.13 & 0.11 \\
\hline \multicolumn{4}{|c|}{ TABLE II } \\
\hline
\end{tabular}

method proposed in our previous work [8] to multivariable case. By using the data of a well designed PRBS type experiments, the identification of static nonlinear part and dynamic linear part is totally decoupled. The identified models can be applied in both steady state and dynamic analysis of human cardio-respiratory responses to exercises.

Noninvasive portable wireless sensors are prone to malfunctions and/or failures (such as, artefacts generated by environmental EMI and disconnection of electrodes). Another purpose of this study is to provide an efficient way to tolerate sensor malfunctions and/or failures based on these identified models.

\section{REFERENCES}

[1] R. Eston, A. Rowlands, and D. Ingledew, "Validity of heart rate, pedometry, accelerometry for predicting the energy cost of children's activities," J Appl Physiol, vol. 84, pp. 362-371, 1998.

[2] M. Blanke, M. Kinnaert, J. Lunze, and M. Staroswiecki, Diagnosis and Fault-Tolerant Control. Berlin: Springer, 2003.

[3] J. Welsman and N. Armstrong, "Daily physical activity and blood lactate indices of aerobic fitness in children," $\mathrm{Br}$. J. Sports Med., vol. 26, no. 7, pp. 228-232, 1992.

[4] D. Dill, "Oxygen used in horizontal and grade walking and running on the treadmill," J Appl Physiol, vol. 20, pp. 19-22, 1965.

[5] B. Franklin, ACSM's Guidelines for Exercise Testing and Prescription. New York: Lippincott Williams \& Wilkins, 2000.

[6] D. Pearce and H. Milhorn, Jr., "Dynamic and steady-state respiratory responses to bicycle exercise," J. Appl. Physiol., vol. 42, no. 6, pp. 959-967, 1977.

[7] B. Whipp, S. Ward, N. Lamarra, J. Davis, and K. Wasserman, "Parameters of ventilatory and gas exchange dynamics during exercise," $J$. Appl. Physiol., vol. 52, no. 6, pp. 1506-1513, 1982.

[8] S. Su, L. Wang, B. Celler, and A. Savkin, "Oxygen uptake estimation in humans during exercise using a Hammerstein model," Ann. Biomed. Eng., vol. 35, no. 11, pp. 1898-1906, 2007.

[9] S. Su, L. Wang, B. Celler, A. Savkin, and Y. Guo, "Identification and control for heart rate regulation during treadmill exercise," IEEE Trans. Biomed. Eng., vol. 54, no. 7, pp. 1238-1246, 2007.

[10] E. Bai, "Decoupling the linear and nonlinear parts in Hammerstein model identification," Automatica, vol. 40, pp. 671-676, 2004.

[11] B. Schlkopf and A. Smola, Learning with kernels. Cambridge: MA: MIT Pres, 2002.

[12] V. Vapnik, Statistical Learning Theory. New York: John Wiley, 1998.

[13] DI Prampero, P. Davies, P. Cerretelli, and R.Margria, "An analysis of $o_{2}$ debt contracted in submaximal exercise," J. Appl. Physiol., vol. 29, pp. 547-551, 1970.

[14] J. Hagberg, R. Hickson, A. Ehsani, and J. Holloszy, "Faster adjustment to and recovery from submaximal exercise in the trained state," J. Appl. Physiol., vol. 48, pp. 218-224, 1980.

[15] A. Hill and H. Lupton, "Muscular exercise, lactic acid and the supply and utilization of oxygen," Q. J. Med., vol. 16, pp. 135-171, 1923. 